\title{
Facilitation of schematic concept formation as a function of two within-schema pretraining modes
}

WILLIAM C. RANKIN and SELBY H. EVANS, Texas Christian University, Fort Worth, Texas 76129

Effects of prior training on schematic concept formation (SCF) were studied in a transfer paradigm employing two types of pretraining experience. One group scaled pairs of patterns from a single schema family; another group encountered the same set of patterns in a task requiring reproduction from memory. Upon transfer to a SCF task, the scaling group exhibited initial superiority. A group that had scaled random stimuli and a group that had no pretraining performed similarly over the first few transfer trials, but at a level below the scaling group and above the reproduction group. Discussion of results centered around a "nonspecific" transfer phenomenon.

Schematic concept formation (SCF) has been defined by Evans (1967) as the development of the ability to assign objects to their corresponding schema families on the basis of the information derived from perceiving the objects. A schema family is a population of objects which can be efficiently described in terms of the same prototype, for example, dogs. The assignment of objects to their appropriate schema families is assumed to be possible without any aid from other sources of information (such as knowledge of results) about the proper categorization.

If only one schema is represented in a set of objects, Evans has theorized, learning of the schema occurs spontaneously when Ss have an opportunity to inspect several examples. When two or more schema families are represented, SCF is supposed to occur. Since SCF depends upon learning the schema, or prototype, of each schema family, it follows that prior opportunities to inspect examples of a relevant schema family would lead to enhanced performance on a subsequent SCF task. The conditions of inspection presumably influence the extent to which the schema is learned. In the present study the effects of two degrees of schema relevance and of two different tasks were investigated as pretraining variables.

If Ss are pretrained with examples of a schema which is then presented as one of the schemata in the subsequent SCF task, they should transfer to that task both the general concept of what constitutes a schema and the specific knowledge of one schema. If the Ss are pretrained with examples of a schema which is not presented in the subsequent SCF task, they can usefully transfer only the general concept of a schema; the specific knowledge of the first schema is no longer relevant. Greater positive transfer would be expected when both general (i.e., nonspecific) and specific pretraining is relevant.

Previous research by Edmonds \& Mueller (1967) demonstrated the effect of nonspecific pretraining with a single schema family in facilitating performance on a subsequent SCF task. The design, however, permitted a conclusion only with regard to nonspecific transfer. The present study permitted a comparison of specific and nonspecific effects in transfer.

The second variable under study in the present experiment was type of pretraining task. Two tasks were used: reproduction and scaling. The reproduction task was methodologically similar to that utilized in a study by Edmonds \& Mueller (1968) in which Ss were to draw from memory the three randomly omitted portions of the pattern they had just viewed. While a scaling task is not known to have been viewed or utilized previously in the role of a pretraining task, it was hypothesized that the requirement of assessing similarity should cause Ss to attend more closely to the schematic aspects of the group of stimuli, with consequent facilitation of early performance on an SCF task where two or more schemata must be discriminated.

\section{SUBJECTS}

The Ss were 60 students enrolled in the introductory psychology courses at Texas Christian University. They were randomly assigned to treatments as they came to the experiment. More than one treatment group were run simultaneously. There were a total of $10 \mathrm{Ss}$ per treatment.

\section{STIMULI}

Patterns were produced by a computer system, VARGUS 9 (Evans \& Mueller, 1966). The VARGUS 9 system produces patterns of numbers randomly sampled from a defined population having specifiable information or redundancy

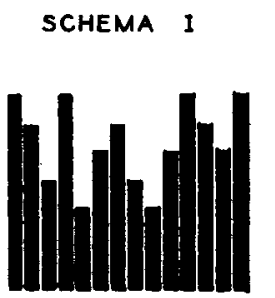

SCHEMA III
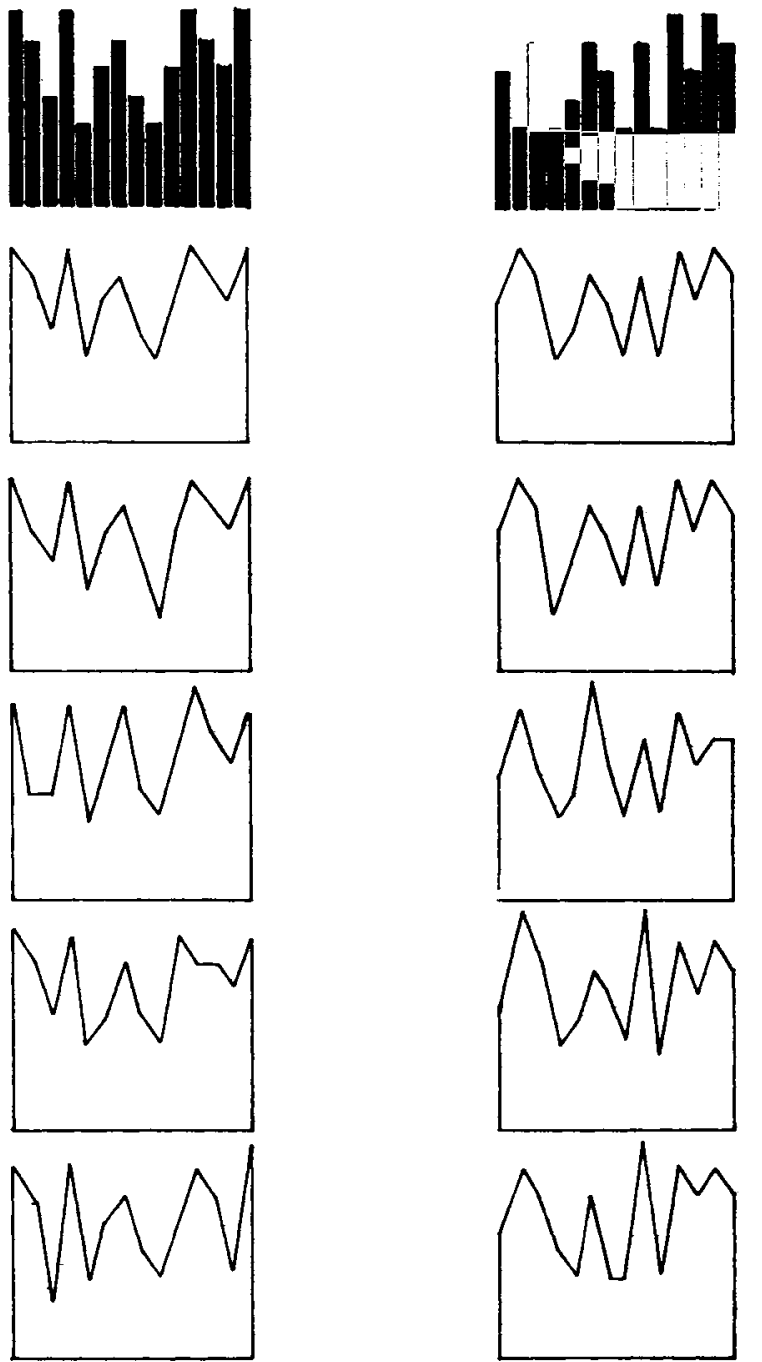

Fig. 1. Two schema prototypes as histoform patterns and examples of their corresponding polygon format together with four instances of deviation from the schema. 
characteristics. The sequence of numbers is mapped into proportional column heights, resulting in patterns aptly described as histoforms. In this experiment the VARGUS 9 histoform patterns were mapped into the shape of closed frequency polygons. Examples of the polygons used are shown in Fig. 1.

Three sets of patterns were required, designated as Schema 1,2 , and 3 . Schemata 1 and 2 were to be discriminated in the transfer task. Schemata 1 and 3 served as the specific and nonspecific schemata, respectively, in the pretraining tasks. All three schemata were $70 \%$ redundant and consisted of a sample of 10 instances each. The samples comprising each schema were constrained to approximate the population distribution of pattern variance (PV) for $70 \%$ redundant schemata. The degree to which a pattern deviates from the schema is measured by PV. Measurement of PV is similar to the calculation of a variance.

\section{TASKS AND PROCEDURE}

Groups making similarity judgments went through four orders of all 45 possible pairs of within-schema comparisons at $10 \mathrm{sec}$ per judgment. In pretraining with the reproduction task, Ss were asked to draw three randomly omitted portions of the patterns from memory; there were five random orders of the 10 stimulus patterns for each schema. The Ss were given $20 \mathrm{sec}$ in the priming phase and $20 \mathrm{sec}$ in the reproduction phase. Patterns in both pretraining tasks were presented in booklet form. Specific treatment groups are detailed below:

S1. Pretraining consisted of scaling Schema 1 similarity.

S3. Pretraining consisted of scaling Schema 3 similarity.

SRAN. Pretraining consisted of scaling random pattern similarity.

R1. Pretraining consisted of Schema 1 reproductions.

R3. Pretraining consisted of Schema 3 reproductions.

NPT. No pretraining was given.

The transfer task followed pretraining after an intertask interval of about $1 \mathrm{~min}$. Ss were told that the task would test their ability to learn to recognize different types of patterns and that they should answer in terms of whether the patterns on each page were examples of the same type of pattern or different types of patterns. The task consisted of looking at pairs of patterns (one pair per page in 10-sec intervals). Since SCF does not require knowledge of results, none was provided throughout the 64 discrimination trials of the transfer task.

It should be noted that the transfer task constituted a discrimination among equal numbers of same-member or different-member pairings of patterns characterizing Schema 1 and Schema 2. Furthermore, the 10 Schema 1 patterns in this task were a completely different sample from that used for all the pretraining groups exposed to Schema 1. Within blocks of eight trials, patterns were semi-randomly grouped so that (1) mean PVs were equal from block to block, and, (2) within each block the random pairings were constrained so as not to exceed a ratio of six same to two different schemata.

\section{RESULTS}

An analysis of the number of correct responses in the first eight trials, where transfer effects should be greatest, showed a significant pretraining group effect $(F=3.186$, df $=5 / 54$, $\mathrm{p}<.05)$. Orthogonal comparisons among treatment means on the first block of eight trials are itemized as follows:

(1) Schema scaling groups vs No pretraining control (SI and $S 3$ vs NPT), significant $(F=4.380, \mathrm{df}=1 / 54, \mathrm{p}<.05)$

(2) Schema scaling groups vs random scaling group (S1 and $S 3$ vs SRAN), significant $(F=5.254, \quad$ df $=1 / 54$, $\mathrm{p}<.05)$.

(3) Schema reproduction groups vs No pretraining control ( $R 1$ and R3 vs NPT), not significant $(F=.993)$.

Although statistically significant differences were not present in the analysis of the groups' performances over all trials in the transfer task, Fig. 2 shows their performance in blocks of 16 trials. Groups S1 and S3 have been combined

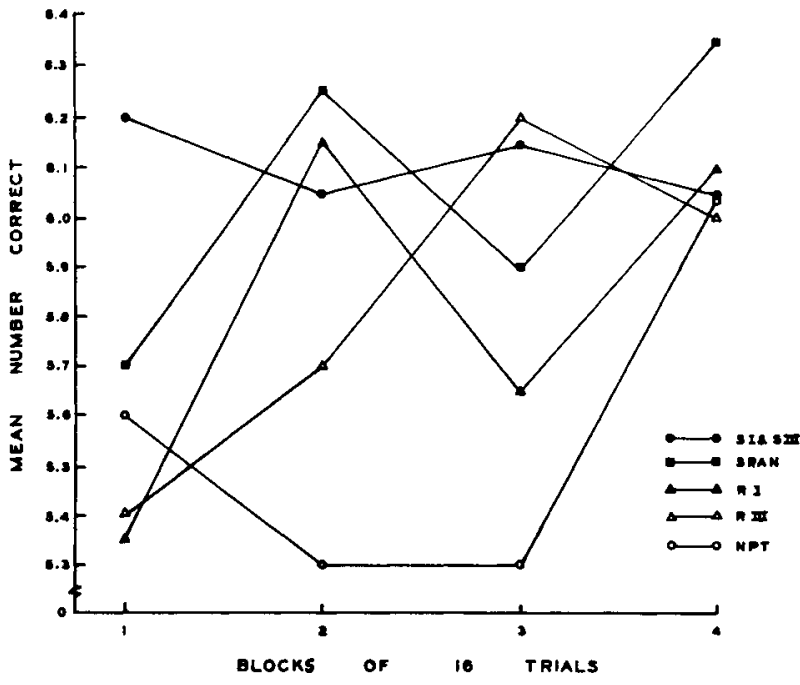

Fig. 2. Performance of the pretraining groups on the betweenschemata discrimination transfer task (in terms of mean number correct per eight trial block).

because they were highly similar. Fig. 2 shows that all groups reached approximately the same level of performance before the end of the test task. The order in which the groups reached this level may be worth noting. The groups with scaling pretraining (S1 and S3) started out at the higher level. Group SRAN, which scaled random stimuli, reached the level next, followed by the two reproduction pretraining Groups (R1 and R3). The group which received no pretraining as a control (NPT) was the last to attain this level. The data presented in Fig. 2 suggest that the effect of pretraining may be indicated not only by the performance on initial trials of the transfer task, but also by the pattern of performance over several blocks of trials.

DISCUSSION

While the groups that made similarity judgments in pretraining exhibited greater transfer than the reproduction groups, there is nothing in the data to suggest that schema relevance had any effect with respect to pretraining task. As suggested by Edmonds \& Mueller (1967), this nonspecifiable transfer could amount to no more than the general notion of what a schema is. It remains a problem, perhaps partly methodological, to design an experiment to demonstrate that prior schema learning will transfer to SCF in an unambiguous fashion.

Although this study was limited to a within-schema scaling task, further experiments should be undertaken employing the similarity judgmental response. In fact, some pilot data suggest that similarity judgments would be a particularly sensitive method of investigating SCF. Experimental evidence, however, is needed to assess the relationship between similarity judgments and the categorical judgments currently used in SCF tasks.

\section{REFERENCES}

EDMONDS, E. M., \& MUELLER, M. R. The role of schemata in perceptual learning. Psychonomic Science, 1967, 8, 239-240.

EDMONDS, E. M., \& MUELLER, M. R. Effects of incidental training and reinforcement on mixed schema learning. Psychonomic Science, $1968,10,75-76$

EVANS, S. H. A brief statement of schema theory. Psychonomic Science, 1967, 8, 87-88.

EVANS, S. H., \& MUELLER, M. R. VARGUS 9: Computed stimuli for schema research. Psychonomic Science, 1966, 6, 511-512. NOTE

1. This research was supported by the Department of Defense, Project THEMIS Contract (DAADO5-68-C-0176), under the Department of the Army to the Institute for the Study of Cognitive Systems through the TCU Research Foundation. 